\title{
Association between SMAD3 gene polymorphisms and osteoarthritis risk: a systematic review and meta-analysis
}

\author{
Jian-qiao Hong ${ }^{\dagger}$, Yang-xin Wang ${ }^{\dagger}$, Si-hao Li, Guang-yao Jiang, Bin Hu, Yu-te Yang, Jia-hong Meng and Shi-gui Yan ${ }^{*}$ (D)
}

\begin{abstract}
Objective: Several studies have been performed to investigate the association between SMAD3 gene polymorphism and osteoarthritis (OA), but the results were inconclusive. This study aims to determine whether SMAD3 polymorphism is associated with risk of $O A$.
\end{abstract}

Method: A comprehensive literature search in PubMed, Embase, and ISI Web of Science for relevant studies was performed. After extracting data from eligible studies, we chose the fixed or random effect model according to the heterogeneity test. Estimation of publication bias and sensitivity analysis were conducted to confirm the stability of this meta-analysis.

Results: In total, 10 studies from 6 articles with 5093 OA patients and 5699 controls were enrolled in this meta-analysis. The combined results revealed significant association between SMAD3 rs12901499 polymorphism and the risk of OA (allele model: OR 1.21, 95\% Cl 1.07-1.38). Subgroup analysis revealed that $\mathrm{G}$ allele increased the risk of OA in Caucasians, but not in Asians (allele model: Caucasians: OR 1.31, 95\% Cl 1.18-1.44; Asians: OR 1.24, 95\% Cl 0.95-1.61). And the pooled results revealed significant association between SMAD3 rs12901499 polymorphism and both knee and hip OA (knee OA: OR 1.18, 95\% Cl 1.04-1.34; hip OA: OR 1.31, 95\% Cl 1.18-1.44).

Conclusion: The current meta-analysis revealed that the G variant of SMAD3 rs12901499 polymorphism increased the risk of OA in Caucasians. Further well-designed studies with larger sample size in different ethnic populations are required to confirm these results.

Keywords: SMAD3, Polymorphism, Osteoarthritis, Meta-analysis

\section{Background}

Osteoarthritis (OA), a late-onset musculoskeletal disease in the elder, is featured by the gradual degradation of articular cartilage with further lesion to the synovium, subchondral bone, or the other joint tissues. The osteoarthritis could cause chronic joint pain with swelling and restricted range of motion through the pathologic process including narrowing of joint space and osteophyte formation [1-3]. It was estimated that over $15 \%$ of the population suffered from OA, and the number tends to be doubled by 2020 due to the increasing elder population $[4,5]$. Though the mechanism of osteoarthritis still has not been fully clarified,

\footnotetext{
* Correspondence: zrjwsj@zju.edu.cn

†Jian-qiao Hong and Yang-xin Wang contributed equally to this work. Department of Orthopaedic Surgery, The Second Affiliated Hospital, Zhejiang University School of Medicine, No.88 Jiefang Road, Hangzhou 310009,
} People's Republic of China

(c) The Author(s). 2018 Open Access This article is distributed under the terms of the Creative Commons Attribution 4.0 International License (http://creativecommons.org/licenses/by/4.0/), which permits unrestricted use, distribution, and reproduction in any medium, provided you give appropriate credit to the original author(s) and the source, provide a link to the Creative Commons license, and indicate if changes were made. The Creative Commons Public Domain Dedication waiver (http://creativecommons.org/publicdomain/zero/1.0/) applies to the data made available in this article, unless otherwise stated. age, sex, obesity, trauma in the joint, environmental factors, and genetic factors $[3,6,7]$.

The SMAD3 (SMAD family member 3 ) gene, located on chromosome $15 \mathrm{q} 22.33$, acts a critical role in the joint homeostasis $[8,9]$. It is known as a downstream mediator in the TGF-B (transforming growth factor-b) signaling pathway which plays a key role in anabolism of chondrocytes [9]. The genetic variations of TGF-B signaling have been reported significant relationship to the OA [10]. In TGF- $\beta$ signaling pathway, SMAD3 translocates into the nucleus and regulates target gene transcription and produces the phenotype in cartilage by interaction with DNA and transcription factors [11]. Several studies have been performed to investigate the association between SMAD3 polymorphisms and OA susceptibility, but the results

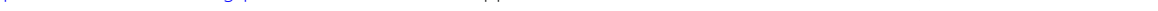


remained unclear. Valdes [12] revealed that the SMAD3 gene rs12901499 polymorphism was associated with hip and knee OA in European populations. However, the results reported by other subsequent studies remain inconsistent and inconclusive [13-17]. In this study, we therefore performed a meta-analysis to evaluate whether the SMAD3 gene polymorphisms are associated with the risk of OA.

\section{Materials and methods}

\section{Literature search strategy}

We conducted a comprehensive literature search using the electronic databases PubMed, Embase, and ISI Web of Science for relevant studies published in English (last search was updated on April 10, 2018). The search strategy was based on the following keywords: "SMAD3" OR "SMAD family member 3") AND ("polymorphism" OR "variant" OR "SNP") AND ("osteoarthritis" OR "OA"). References of clinical trials and review articles were also searched manually for additional articles. All the literature search was performed according to the Preferred Reporting Items for Systematic Reviews and Meta-Analyses (PRISMA) guidelines (Additional file 1: Table S1) [18].

\section{Inclusion criteria}

Two researchers screened the relevant investigations and further determined the eligible studies which met the following inclusion criteria: (1) case-control or cohort design; (2) evaluating the association between SMAD3 polymorphisms and knee/hip OA susceptibility; (3) patients with OA were diagnosed based on clinical manifestation and radiographic findings, or received total joint arthroplasty because of primary OA; and (4) enough data on genotype or allele frequency for calculation of odds ratio (OR) and corresponding 95\% confidence interval (CI). The animal model research, review, case reports, or the studies without sufficient data were excluded. If several articles reported findings for repeated study populations, we only selected the most recent study or the one with the largest sample size.

\section{Data extraction}

For each eligible study, two independent investigators extracted the following data: first author's name, publication year, country and ethnicity of study population, study design, OA sites, sample size, demographics of enrolled subjects, genotyping method, studied polymorphisms, and genotype distributions.

\section{Quality assessment}

According to the Newcastle-Ottawa Quality Assessment Scale (NOS) [19], the quality score of each study was based on three categories: selection ( 4 items, 1 point each), comparability ( 1 item, up to 2 points), and exposure/outcome ( 3 items, 1 point each). Each

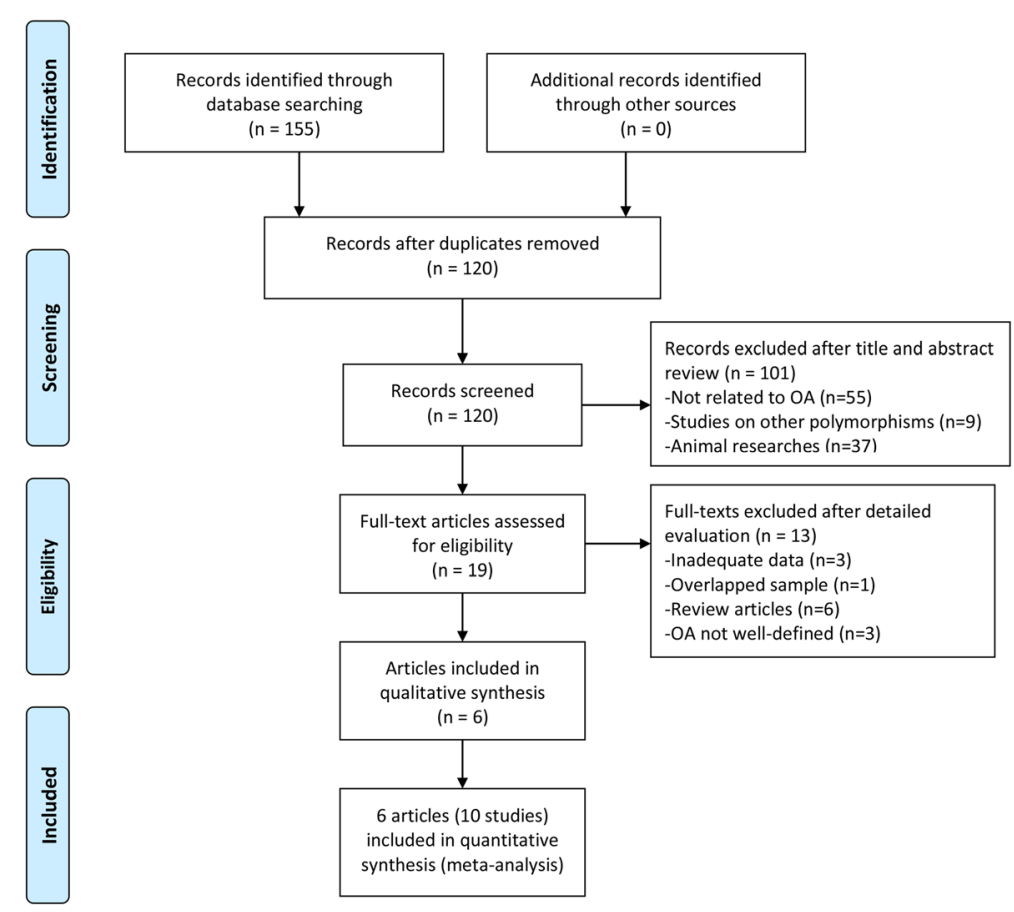

Fig. 1 Flow chart of the study selection process 
Table 1 Characteristics of included studies

\begin{tabular}{|c|c|c|c|c|c|c|c|c|c|c|}
\hline \multirow[t]{2}{*}{ Study } & \multirow[t]{2}{*}{ Year } & \multirow[t]{2}{*}{ Country } & \multirow[t]{2}{*}{ Ethnicity } & \multirow[t]{2}{*}{ Design } & \multirow[t]{2}{*}{ OA site } & \multirow{2}{*}{$\begin{array}{l}\text { Genotyping } \\
\text { method }\end{array}$} & \multicolumn{2}{|c|}{ Sample size (F/M) } & \multirow[t]{2}{*}{ HWE } & \multirow[t]{2}{*}{ NOS } \\
\hline & & & & & & & $\overline{\mathrm{OA}}$ & Control & & \\
\hline Valdes-Discovery set & 2010 & UK & Caucasian & $\mathrm{CC}$ & Knee, hip & Allele-specific PCR & 477 (NA) & 520 (NA) & Y & 7 \\
\hline Valdes-Nottingham & 2010 & UK & Caucasian & $\mathrm{CC}$ & Knee, hip & Allele-specific PCR & 2014 (NA) & 733 (NA) & Y & 7 \\
\hline Valdes-Chingford & 2010 & UK & Caucasian & Cohort & Knee, hip & Allele-specific PCR & $317(317 / 0)$ & $488(488 / 0)$ & Y & 9 \\
\hline Valdes-Hertfordshire & 2010 & UK & Caucasian & Cohort & Knee & Allele-specific PCR & 167 (NA) & 867 (NA) & Y & 8 \\
\hline Valdes-Estonia & 2010 & Estonia & Caucasian & Cohort & Knee & Allele-specific PCR & $68(N A)$ & 449 (NA) & Y & 8 \\
\hline Jiang & 2013 & China & Asian & CC & Knee & PCR-RFLP & $232(159 / 73)$ & $236(91 / 145)$ & Y & 7 \\
\hline Su & 2015 & China & Asian & $\mathrm{CC}$ & Knee & PCR-RFLP & $518(328 / 190)$ & $468(261 / 207)$ & Y & 7 \\
\hline Sharma & 2017 & India & Asian & CC & Knee & Allele-specific PCR & $450(230 / 220)$ & $458(234 / 224)$ & Y & 8 \\
\hline Zhang & 2018 & China & Asian & CC & Knee & Allele-specific PCR & $350(99 / 251)$ & $400(110 / 210)$ & Y & 8 \\
\hline Zhong & 2018 & China & Asian & $\mathrm{CC}$ & Hip & Allele-specific PCR & $500(260 / 240)$ & $1080(580 / 500)$ & Y & 8 \\
\hline
\end{tabular}

CC case-control, F female, $M$ male, NA number of each gender not available, HWE Hardy-Weinberg equilibrium, NOS Newcastle-Ottawa Quality Assessment Scale

study scored from 0 point (worst) to 9 points (best), and scored 6 or less were classified as low quality, whereas studies scoring 7 or higher were defined as high quality.

\section{Statistical analysis}

All statistical analyses were conducted with STATA version 12.0 (STATA Corporation, College Station, TX, USA), and $p$ value $<0.05$ was considered significant except for the $I^{2}$ statistic. To assess the correlation between SMAD3 polymorphisms and OA susceptibility, we calculated pooled ORs with 95\% CI and analyzed five genetic models: allele model, dominant model, recessive model, homozygous model, and heterozygous model.

Heterogeneity between studies was measured using $Q$ and $I^{2}$ statistics [20]. If $I^{2}>50 \%$ and $p$ value of $Q$ statistic $<0.10$, the DerSimonian-Laird random effect model was applied to calculate pooled ORs and 95\% CIs [21].
Otherwise, a fixed effect model was used as the pooling method [22].

Subgroup analysis was conducted by ethnicity, OA site. Sensitivity analysis was also performed by removing individual study sequentially in order to evaluate the stability of pooled results. We evaluated the publication bias by funnel plot and Egger's regression test.

\section{Results}

\section{Study selection}

Figure 1 presented the selection process and reasons for exclusion. One hundred fifty-five articles were retrieved totally from a systematic literature search, 35 articles were removed because of duplications, and 101 articles were excluded after review of title and abstract; only 19 full-text articles remained for further evaluation. Subsequently, 4 articles were excluded because of inadequate data, 1 article was excluded due to overlapped

Table 2 Genotype and allele distributions of SMAD3 rs12901499 polymorphism in the included studies

\begin{tabular}{|c|c|c|c|c|c|c|c|c|c|c|c|}
\hline \multirow[t]{3}{*}{ Study } & \multicolumn{6}{|c|}{ Genotype distribution } & \multicolumn{4}{|c|}{ Allele distribution } & \multirow[t]{3}{*}{ Association findings } \\
\hline & \multicolumn{3}{|l|}{$\mathrm{OA}$} & \multicolumn{3}{|c|}{ Control } & \multicolumn{2}{|l|}{$\mathrm{OA}$} & \multicolumn{2}{|c|}{ Control } & \\
\hline & $\mathrm{AA}$ & $A G$ & GG & $\mathrm{AA}$ & $A G$ & GG & A & G & A & G & \\
\hline Valdes-Discovery set & NA & NA & NA & NA & NA & NA & 419 & 635 & 489 & 551 & G allele $\uparrow$ \\
\hline Valdes-Nottingham & NA & NA & NA & NA & NA & NA & 1788 & 2336 & 698 & 768 & G allele $\uparrow$ \\
\hline Valdes-Chingford & NA & NA & NA & NA & NA & NA & 316 & 388 & 477 & 499 & NS \\
\hline Valdes-Hertfordshire & NA & NA & NA & NA & NA & NA & 134 & 200 & 779 & 955 & NS \\
\hline Valdes-Estonia & NA & NA & NA & NA & NA & NA & 67 & 79 & 481 & 417 & NS \\
\hline Jiang & 47 & 141 & 25 & 114 & 83 & 23 & 235 & 191 & 311 & 129 & G allele $\uparrow$, GG genotype $\uparrow$ \\
\hline Su & 142 & 247 & 129 & 116 & 228 & 124 & 531 & 505 & 460 & 476 & NS \\
\hline Sharma & 85 & 70 & 75 & 90 & 92 & 52 & 240 & 220 & 272 & 196 & G allele $\uparrow$, GG genotype $\uparrow$ \\
\hline Zhang & 82 & 173 & 91 & 81 & 202 & 111 & 337 & 355 & 364 & 424 & GG genotype $\downarrow$ \\
\hline Zhong & 10 & 200 & 290 & 20 & 610 & 450 & 220 & 780 & 650 & 1510 & G allele $\uparrow$, GG genotype $\uparrow$ \\
\hline
\end{tabular}

NA data not available, $\uparrow / \downarrow$ increase/decrease the risk of OA, NS not significant 
Table 3 Pooled results on the association between SMAD3 rs12901499 polymorphism and OA risk

\begin{tabular}{|c|c|c|c|c|c|c|c|}
\hline \multirow[t]{2}{*}{ Genetic model } & \multirow[t]{2}{*}{ Sub-group } & \multirow{2}{*}{$\begin{array}{l}\text { No. of } \\
\text { studies }\end{array}$} & \multicolumn{2}{|c|}{ Test of association } & \multirow{2}{*}{$\begin{array}{l}\text { Statistical } \\
\text { model }\end{array}$} & \multicolumn{2}{|c|}{ Test of heterogeneity } \\
\hline & & & $\overline{\mathrm{OR}(95 \% \mathrm{Cl})}$ & $p$ & & $\bar{P}(\%)$ & $p$ \\
\hline \multirow[t]{7}{*}{ Allele model (G vs. A) } & Overall & 10 & $1.21(1.07-1.38)$ & 0.003 & Random & 75.4 & 0.000 \\
\hline & \multicolumn{7}{|l|}{ Ethnicity } \\
\hline & Asian & 5 & $1.24(0.95-1.61)$ & 0.115 & Random & 88.7 & 0.000 \\
\hline & Caucasian & 5 & $1.20(1.11-1.31)$ & 0.000 & Fixed & 0.0 & 0.891 \\
\hline & \multicolumn{7}{|l|}{ OA site } \\
\hline & Knee OA & 9 & $1.18(1.04-1.34)$ & 0.011 & Random & 67.1 & 0.002 \\
\hline & Hip OA & 4 & $1.31(1.18-1.44)$ & 0.000 & Fixed & 39.9 & 0.172 \\
\hline
\end{tabular}

samples, and 8 review articles were removed; 3 articles studied generalized OA, temporomandibular joint OA, and spinal OA, respectively, and were not included because the OA patients included by those studies were not well defined.

Finally, 6 articles met our inclusion criteria [12-17]. One article provided by Valdes included 5 different study populations; these 5 studies were analyzed independently. Therefore, 10 independent studies from 6 articles were included in our meta-analysis.

\section{Characteristics of included studies}

Table 1 shows the main characteristics of included studies, and Table 2 presents the genotype and allele distributions of the SMAD3 rs12901499 polymorphism. A total of 5093 OA patients and 5699 controls were included in this study, which involved 5 Caucasian and 5
Asian populations. Patients diagnosed with OA were recruited according to clinical and radiographic results or ascertained by total joint arthroplasty. The genotype distribution of the control group showed conformation to Hardy-Weinberg equilibrium in all the included studies. As for the sites of OA, 9 studies examined knee $\mathrm{OA}$, and 4 studies examined hip OA. Regarding the NOS scale, the quality of all the included studies was fairly high (Additional file 2: Table S2). Eventually, all the 10 studies from the articles as stated above were included in the meta-analysis for further research.

\section{Association between SMAD3 rs12901499 polymorphism and $O A$ susceptibility}

Table 3 and Fig. 2 summarize the meta-analysis results on the association between SMAD3 rs12901499 polymorphism and risk of OA. Because genotype distribution data

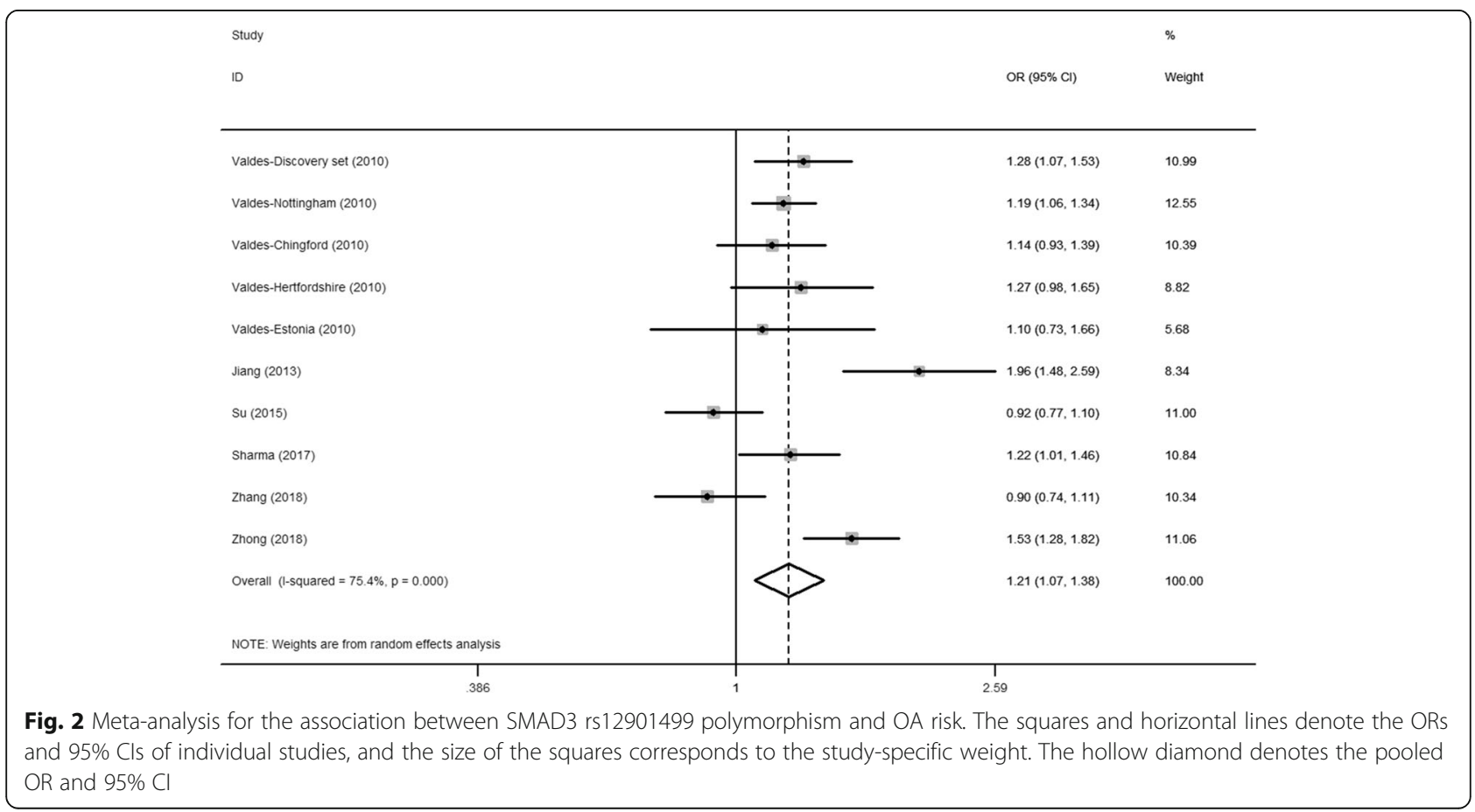


Table 4 Pooled results on the association between SMAD3 rs12901499 polymorphism and OA risk in Asians

\begin{tabular}{|c|c|c|c|c|c|c|}
\hline \multirow[t]{2}{*}{ Genetic model } & \multirow{2}{*}{$\begin{array}{l}\text { No. of } \\
\text { studies }\end{array}$} & \multicolumn{2}{|c|}{ Test of association } & \multirow{2}{*}{$\begin{array}{l}\text { Statistical } \\
\text { model }\end{array}$} & \multicolumn{2}{|c|}{ Test of heterogeneity } \\
\hline & & OR $(95 \% \mathrm{Cl})$ & $p$ & & $P(\%)$ & $p$ \\
\hline Allele model (G vs. A) & 5 & $1.28(0.87-1.88)$ & 0.203 & Random & 90.1 & 0.000 \\
\hline Dominant model ( $G G+A G$ vs. $A A)$ & 5 & $1.18(0.70-2.00)$ & 0.529 & Random & 90.2 & 0.000 \\
\hline Recessive model (GG vs. AG+AA) & 5 & $1.29(0.90-1.86)$ & 0.166 & Random & 85.3 & 0.000 \\
\hline Homozygote model (GG vs. AA) & 5 & $1.21(0.83-1.77)$ & 0.324 & Random & 70.6 & 0.009 \\
\hline Heterozygote model (AG vs. AA) & 5 & $1.06(0.56-1.99)$ & 0.857 & Random & 92.3 & 0.000 \\
\hline
\end{tabular}

was not reported by Valdes, only allele model was analyzed in the overall population and European population. Overall, the combined results revealed a significant association between SMAD3 rs12901499 polymorphism and the risk of OA (allele model: OR 1.21, 95\% CI 1.07-1.38); (Table 3; Fig. 2).

When we divided the participants according to ethnicity, G allele was associated with increased risk of OA in Caucasian rather than in Asians (allele model: Caucasian: OR 1.31, 95\% CI 1.18-1.44; Asian: OR 1.24, 95\% CI $0.95-1.61$ ) (Table 3). What is more, stratification by OA site showed that SMAD3 rs12901499 polymorphism was significantly associated with the risk of both knee OA and hip OA (knee OA: OR 1.18, 95\% CI 1.04-1.34; hip OA: OR 1.31, 95\% CI 1.18-1.44) (Table 3).

For Asian subgroup, other genetic models were also analyzed (allele model: OR 1.28, 95\% CI 0.87-1.88; dominant model: OR 1.18, 95\% CI 0.70-2.00; recessive model: OR 1.29, 95\% CI 0.90-1.86; homozygote model: OR 1.21, 95\% CI 0.83-1.77; heterozygote model: OR 1.06, 95\% CI 0.56-1.99) (Table 4).

\section{Sensitivity and publication bias analysis}

With the aid of funnel plots and Egger's test (Table 3; $p$ egger $=0.692$ ), we find no significant publication bias (Fig. 3). Furthermore, by using sensitivity analysis (Fig. 4), the combined results remained stable after

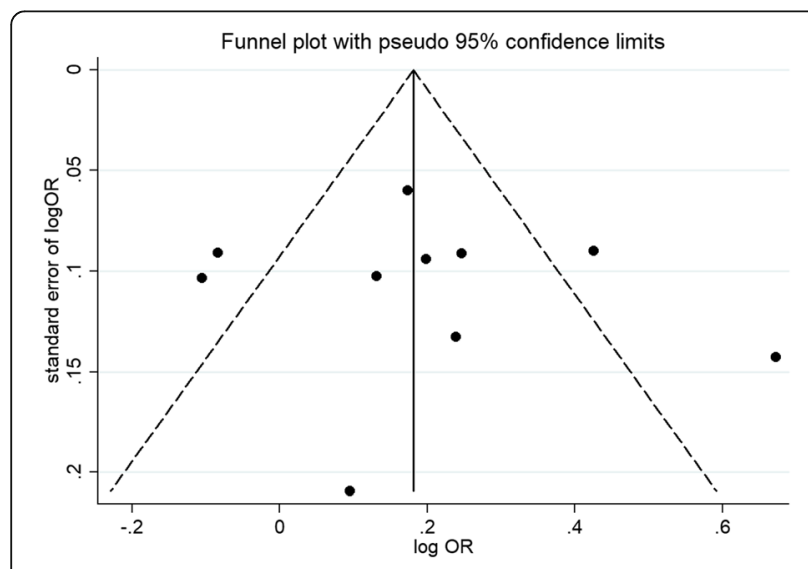

Fig. 3 Funnel plot removing individual studies. The robustness of summarized estimate was shown by the data above.

\section{Discussion}

OA, known as a degenerative disease in the aging population, is the most universal cause of joint disease which could finally result in physical disability [23]. Although $\mathrm{OA}$ is considered as a multifactorial disease, genetic factors are reported as vital determinants in the pathogenesis of this disorder [6, 7]. Enormous attention has been paid to the association between gene SNPs and risk of osteoarthritis, and SMAD3 SNP rs12901499 was studied by several researchers. In different studies, the results ranged from no association to the strong linkage between the SNPs and the disorder $[12,14,16,17]$. The inconsistent findings on the associations between $\mathrm{OA}$ and SMAD3 rs12901499 polymorphism in Asians from relatively underpowered studies above may be attributed to factors like small sample size and different population. So we conducted this systematic review and metaanalysis to draw a more definitive conclusion.

To obtain compelling evidence of the linkage between SMAD3 rs12901499 polymorphism and risk of OA, we enrolled a total of 5093 OA patients and 5699 controls from 10 studies in this meta-analysis. The pooled results showed there is an association between $\mathrm{OA}$ and rs12901499 polymorphism in the overall population. And subgroup analysis stratified by ethnicity demonstrated that $\mathrm{G}$ allele increased the risk of OA in Caucasian. While in Asians, no association was found between SMAD3 rs12901499 polymorphism and OA risk. The results seem intriguing that there is association in the overall population but without positive result in Asian. It could be probably statistically insufficient when the majority of cases of the study were originated from Caucasian (6100/10792). And one other possibility may relate to the different expression patterns of Smad3 in non-homogeneous ethnic populations.

Sensitivity analysis and bias estimation warrant the stability of our meta-analysis.

Based on the present analysis, we propose that patients harboring the G allele of SMAD rs12901499 polymorphism experience an increased susceptibility to OA in Caucasian, though the mechanism underlying the association between 


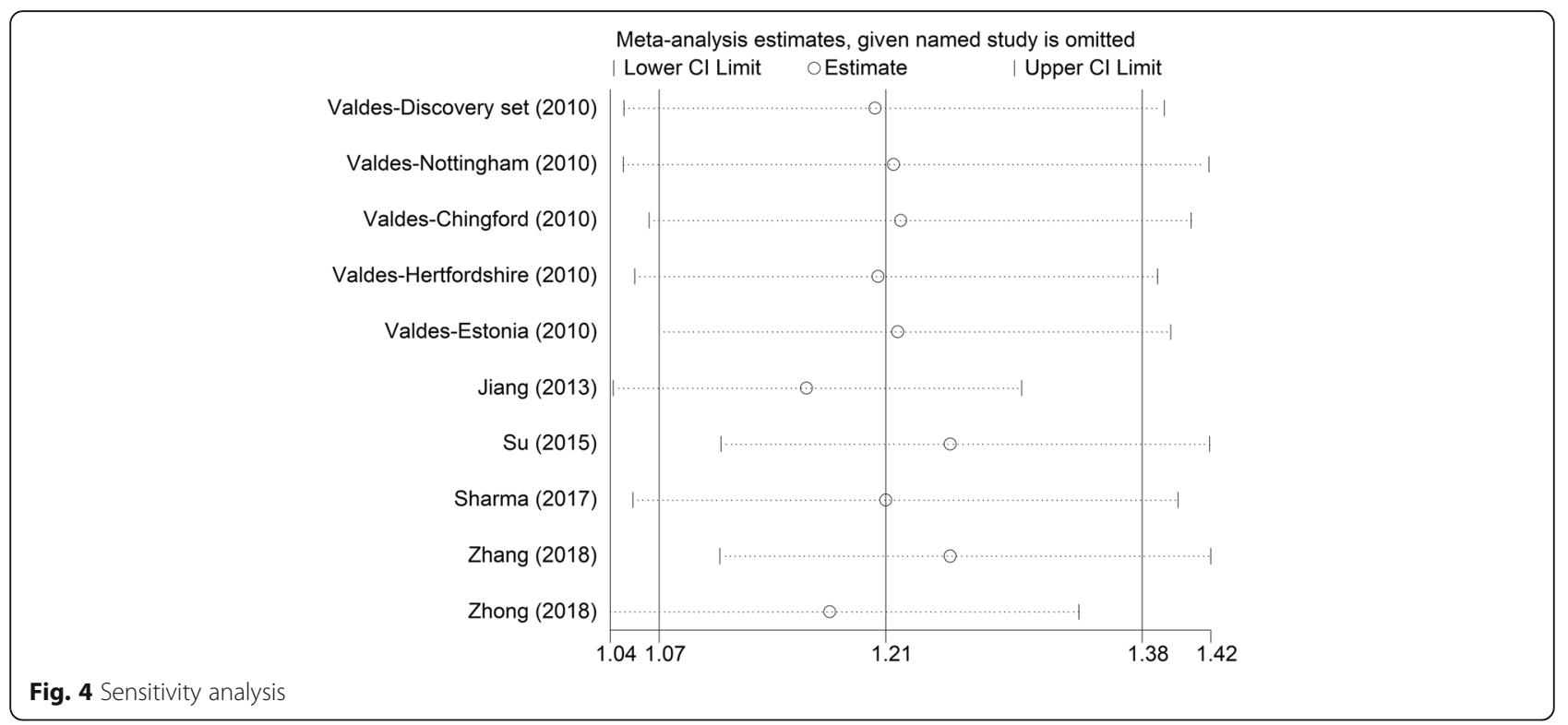

SMAD3 rs12901499 polymorphism and osteoarthritis is temporarily unknown. It was reported that SMAD2/ 3 molecules, known as a part of the TGF-B signaling, have been linked to the chondrocyte anabolism. Previous study showed mutation of SMAD3 could lead to lower expression of type II collagen [17]. Through an in vivo study, Wu et al. found that loss of Smad3 could enhance the BMP signaling to induce articular chondrocytes hypertrophy and lose its normal phenotype [24]. Further functional studies are required to identify the role of SNP rs12901499 in OA susceptibility.

The meta-analysis presented does have some limitations. Firstly, the genotype distribution data was not available in Valdes' study and only allele model among overall population was analyzed to assess the association. Secondly, OA was considered as a multifactorial disease; however, the interactions between the gene and environment were not fully addressed in this meta-analysis, which may magnify the role of Smad3 polymorphism in osteoarthritis. Thirdly, heterogeneity, which comes from different designation about interventions, participants, or outcomes in amount of studies, was inevitably existed though partially addressed by subgroup analysis stratified by ethnic groups and OA sites. Fourthly, since the data is not completely available, the subgroup analysis stratified by every potential confounders in including BMI, age, and gender could not be performed, which may lead to relatively inaccurate pooled results. Last, though there was no obvious publication bias revealed by funnel plot and Egger's test, the selection bias could not be completely removed because only studies published in English were included.

\section{Conclusion}

In conclusion, the present meta-analysis demonstrated that the G variant of SMAD3 rs12901499 polymorphism increased the risk of $\mathrm{OA}$ in Caucasians. By contrast, SMAD3 rs12901499 polymorphism was not associated with OA risk in Asians. Due to the limitations of our study, further well-designed studies with larger sample size in different ethnic populations should be performed to confirm these results.

\section{Additional files}

Additional file 1: Table S1. PRISMA checklist. (DOC 63 kb)

Additional file 2: Table S2. Results of quality assessment for the included studies using the Newcastle-Ottawa Scale. (DOCX 21 kb)

\section{Abbreviations}

OA: Osteoarthritis; OR: Odds ratio; SMAD3: SMAD family member 3; SNP: Single nucleotide polymorphisms; TGF: Transforming growth factor

\section{Acknowledgements}

This study was supported by grants from Zhejiang Province Natural Science Foundation (NO.LQ18H060001), Zhejiang Province medical and health project (NO.2018269731), and Zhejiang Province Chinese medicine project (NO. 2015ZB028).

Availability of data and materials

Please contact the author for data requests.

\section{Authors' contributions}

$\mathrm{JQH}$ and YXW contributed to the design of the study and retrieval of the literatures, extracted and analyzed the data, and wrote the manuscript. SHL contributed to the retrieval of the literatures and extracted and analyzed data. GYJ contributed to the search strategies and extracted the data. $\mathrm{BH}$ participated in the statistical analysis. YTY and JHM helped to screen the manuscript and to review the result of statistical analysis. SGY participated in its design and coordination and helped to review the manuscript. All authors read and approved the final manuscript. 


\section{Ethics approval and consent to participate}

This section is not applicable for our study.

\section{Consent for publication}

This section is not applicable for our study.

\section{Competing interests}

The authors declare that they have no competing interests.

\section{Publisher's Note}

Springer Nature remains neutral with regard to jurisdictional claims in published maps and institutional affiliations.

Received: 5 June 2018 Accepted: 4 September 2018

Published online: 12 September 2018

\section{References}

1. Brandt KD, Dieppe P, Radin EL. Etiopathogenesis of osteoarthritis. Rheum Dis Clin N Am. 2008;34(3):531-59.

2. Brown KA, Pietenpol JA, Moses HL. A tale of two proteins: differential roles and regulation of Smad2 and Smad3 in TGF-beta signaling. J Cell Biochem. 2007;101(1):9-33.

3. Neogi T, Zhang Y. Epidemiology of osteoarthritis. Rheum Dis Clin N Am. 2013;39(1):1-19.

4. Lawrence RC, et al. Estimates of the prevalence of arthritis and other rheumatic conditions in the United States. Part II. Arthritis Rheum. 2008; 58(1):26-35

5. Guccione AA, et al. The effects of specific medical conditions on the functional limitations of elders in the Framingham Study. Am J Public Health. 1994;84(3):351-8.

6. Cooper $\mathrm{C}$, et al. Risk factors for the incidence and progression of radiographic knee osteoarthritis. Arthritis Rheum. 2000;43(5):995-1000.

7. Vaughan MW, et al. Perceived community environmental factors and risk of five-year participation restriction among older adults with or at risk of knee osteoarthritis. Arthritis Care Res (Hoboken). 2017:69(7):952-8.

8. Li Y, et al. SMAD3 regulates follicle-stimulating hormone synthesis by pituitary gonadotrope cells in vivo. J Biol Chem. 2017;292(6):2301-14.

9. van der Kraan PM, et al. TGF-beta signaling in chondrocyte terminal differentiation and osteoarthritis: modulation and integration of signaling pathways through receptor-Smads. Osteoarthr Cartil. 2009;17(12):1539-45.

10. Finnson KW, et al. TGF-b signaling in cartilage homeostasis and osteoarthritis. Front Biosci (Schol Ed). 2012:4:251-68.

11. Finnson $\mathrm{KW}$, et al. Endoglin differentially regulates TGF-beta-induced Smad2/3 and Smad1/5 signalling and its expression correlates with extracellular matrix production and cellular differentiation state in human chondrocytes. Osteoarthr Cartil. 2010:18(11):1518-27.

12. Valdes AM, et al. Genetic variation in the SMAD3 gene is associated with hip and knee osteoarthritis. Arthritis Rheum. 2010;62(8):2347-52.

13. Liying J, et al. A SMAD3 gene polymorphism is related with osteoarthritis in a Northeast Chinese population. Rheumatol Int. 2013;33(7):1763-8.

14. Su SL, et al. Gene-gene interactions between TGF-beta/Smad3 signalling pathway polymorphisms affect susceptibility to knee osteoarthritis. BMJ Open. 2015;5(6):e007931.

15. Sharma AC, et al. Association between single nucleotide polymorphisms of SMAD3 and BMP5 with the risk of knee osteoarthritis. J Clin Diagn Res. 2017;11(6):Gc01-gc04.

16. Zhang L, et al. Association between SMAD3 gene rs12901499 polymorphism and knee osteoarthritis in a Chinese population. J Clin Lab Anal. 2018:32:e22383.

17. Zhong F, et al. Genetic variation of SMAD3 is associated with hip osteoarthritis in a Chinese Han population. J Int Med Res. 2018:46:1178-86.

18. Moher D, et al. Preferred reporting items for systematic reviews and meta-analyses: the PRISMA statement. BMJ. 2009:339:b2535.

19. Stang A. Critical evaluation of the Newcastle-Ottawa scale for the assessment of the quality of nonrandomized studies in meta-analyses. Eur J Epidemiol. 2010;25(9):603-5.

20. Bowden J, et al. Quantifying, displaying and accounting for heterogeneity in the meta-analysis of RCTs using standard and generalised Q statistics. BMC Med Res Methodol. 2011;11:41

21. DerSimonian R, Laird N. Meta-analysis in clinical trials. Control Clin Trials. 1986;7(3):177-88.
22. Mantel N, Haenszel W. Statistical aspects of the analysis of data from retrospective studies of disease. J Natl Cancer Inst. 1959:22(4):719-48.

23. Arden N, Nevitt MC. Osteoarthritis: epidemiology. Best Pract Res Clin Rheumatol. 2006;20(1):3-25.

24. Wu Q, et al. Induction of an osteoarthritis-like phenotype and degradation of phosphorylated Smad3 by Smurf2 in transgenic mice. Arthritis Rheum. 2008:58(10):3132-44

\section{Ready to submit your research? Choose BMC and benefit from:}

- fast, convenient online submission

- thorough peer review by experienced researchers in your field

- rapid publication on acceptance

- support for research data, including large and complex data types

- gold Open Access which fosters wider collaboration and increased citations

- maximum visibility for your research: over $100 \mathrm{M}$ website views per year

At $\mathrm{BMC}$, research is always in progress.

Learn more biomedcentral.com/submissions 